\title{
LAS MISIONES CULTURALES: UN PROYECTO DE EDUCACIÓN Y SALUD EN EL MEDIO RURAL MEXICANO DEL SIGLO XX
}

\author{
Lucía Martínez Moctezuma \\ Universidad Autónoma del Estado de Morelos. México \\ luciamm@uaem.mx
}

\section{RESUMEN}

Una vez terminado el periodo de guerra civil en México (1910-1917) se implementó una vasta campaña de educación higiénica que identificó la influencia de la escuela para difundir diferentes ideas sobre los beneficios de la vacunación y el cuidado de la salud del ciudadano. En este trabajo, me propongo dar cuenta de la práctica de las Misiones Culturales que llegarán a las comunidades rurales para desarrollar una cultura física y educar a los ciudadanos para combatir enfermedades y proteger su salud. En este cambio se percibe una transformación del proyecto educativo mexicano, a saber, la sustitución del modelo escolar europeo que privilegia el entorno urbano desde 1880, por otro norteamericano en las primeras décadas del siglo XX, que atiende a la comunidad rural con el apoyo de un equipo de trabajo en el que participan inspectores, directores, profesores de diferentes especialidades, médicos, enfermeras, padres de familia y la comunidad rural en su conjunto.

Palabras clave: Educación. Salud. Proyecto educativo rural. Misiones culturales. México.

\section{CULTURAL MISSIONS: A PROJECT OF EDUCATION AND HEALTH IN THE MEXICAN RURAL OF THE 20TH CENTURY}

\begin{abstract}
After the period of the civil war in Mexico (1910-1917), a vast hygiene education campaign was implemented that identified the influence of the school in disseminating different ideas about the benefits of vaccination and health care for the citizen. In this work, I intend to give an account of the practice of Cultural Missions that will reach rural communities to develop a physical culture and educate citizens to fight diseases and protect health. In this change we see a transformation of the Mexican educational project, namely, the replacement of the European school model that privileges the urban environment since 1880, by another that is North American in the first decades of the twentieth century, which serves the rural community with the support of a working team in which inspectors, principals, teachers of different specialties, doctors, nurses, parents and the rural community as a whole participate.
\end{abstract}

Keywords: Education. Health. Rural education Project. Cultural missions. Mexico. 


\title{
MISSÃO CULTURAL: UM PROJETO DE EDUCAÇÃO E SAÚDE NAS ÁREAS RURAIS MEXICANOS DO SÉCULO XX
}

\begin{abstract}
RESUMO
Uma vez terminado o período de guerra civil no México (1910-1917) foi implementada uma ampla campanha de educação para a saúde que identificou a influência das diferentes escolas para espalhar ideias sobre os benefícios da vacinação e os cuidados de saúde dos cidadãos. Neste artigo, pretendo explicar a prática das Missões Culturais que irá atingir as comunidades rurais para desenvolver a cultura física e educar os cidadãos para combater a doença e proteger a saúde. Nesta mudança é percebida uma transformação do projeto educativo mexicano, ou seja, a substituição do modelo de escola europeia que favorece o ambiente urbano desde 1880, por outro americano nas primeiras décadas do século XX, servindo a comunidade rural com o apoio de uma equipe de trabalho em que participam inspetores, diretores, professores de diferentes especialidades, médicos, enfermeiros, pais e da comunidade rural como um todo.
\end{abstract}

Palavras chave: Educação. Saúde. Projeto de educação rural. Missões culturais. México.

\section{MISSIONS CULTURELS: UN PROJET D'ÉDUCATION ET SANTÉ DANS LES ZONES RURALES MEXICAINS DU XXE SIÈCLE}

\section{RÉSUMÉ}

Achevée la période de la guerre civile au Mexique (1910-1917) une vaste campagne d'éducation sanitaire a été mise en œuvre qui a identifié l'influence de l'école pour diffuser des idées sur les avantages de la vaccination et la santé des citoyens. Dans cet article, je me propose de rendre compte de la pratique des Missions Culturelles qui atteindront les communautés rurales à développer la culture physique et d'éduquer les citoyens à lutter contre les maladies et protéger leur santé. Dans ce changement, il existe une transformation du projet éducatif mexicain, à savoir le remplacement perçu du modèle de l'école européenne qui favorise l'environnement urbain depuis 1880, par un autre américain dans les premières décennies du XXe siècle, au service de la communauté rurale avec le soutien de une équipe participant des inspecteurs, directeurs, professeurs de différentes spécialités, les médecins, les infirmières, les parents et la communauté rurale dans son ensemble.

Mots-clés: Éducation. La santé. Projet d'éducation rurale. Missions culturelles. Mexique.

\section{INTRODUCCIÓN}

El proyecto modernizador del gobierno de Porfirio Díaz (1877-1910) buscó transformar el país en una nación de ciudadanos trabajadores, sanos y vigorosos que fueran el reflejo del progreso y la prosperidad de la época. Para esto se expidieron reglamentos, códigos penales y de salud, se construyeron grandes obras de infraestructura sanitaria, caminos, líneas férreas y se 
tomaron una serie de medidas contra la insalubridad y la falta de higiene para combatir enfermedades endémicas y epidémicas como la viruela ${ }^{1}$ que beneficieron sobre todo al medio urbano.

La fase armada de la Revolución Mexicana (1910-1917) marcó el fin de este período: una década de constantes cambios y defensa de diversas demandas políticas, sociales y agrarias pero también de largos periodos de violencia e insalubridad que afectó al 70\% de los 15 millones de mexicanos que vivían en pequeñas poblaciones rurales (pueblos, haciendas, ranchos) con menos de 2500 habitantes, desprovistos además de infrestructura sanitaria, y medios de comunicación. Una vez terminado este periodo de guerra civil, hubo interés de parte de las autoridades por controlar las epidemias aplicando vacunas de manera obligatoria y a nivel nacional, que despertó inquietud entre los pobladores ante la presencia de enfermeras y médicos que llegaban a sus comunidades con la consigna de aplicarlas de manera obligatoria ${ }^{2}$. En este marco se hizo necesario implementar una vasta campaña de educación higiénica que identificó a la escuela como uno de los espacios privilegiados para difundir las ideas en torno a los beneficios de la vacunación y el cuidado de la salud del ciudadano. Es en este sentido que el objetivo de mi trabajo es dar cuenta de esta práctica novedosa que va más allá del espacio físico de la escuela porque el nuevo proyecto educativo, las Misiones Culturales, se orientará hacia las comunidades rurales para educar, desarrollar una cultura física, combatir las enfermedades y proteger la salud de los ciudadanos. En este cambio se percibe una transformación del proyecto educativo mexicano, a saber, la sustitución del modelo escolar europeo que privilegia el entorno urbano desde 1880, por otro norteamericano en las primeras décadas del siglo XX, que atiende a la comunidad rural con el apoyo de un equipo de trabajo en el que participan inspectores, directores,

\footnotetext{
${ }^{1}$ Hacia 1880 se habían registrado cerca de 20 epidemias y, a decir, del médico Domingo Orvañanos (1899), se trataba de una enfermedad que producía la muerte, originaba la perdida de la vista y siempre, desfiguraba la fisionomía de las personas a causa de las cicatrices que dejaba, por esto, desde 1841 se había intentado contener la enfermedad con la aplicación de la vacuna de brazo a brazo y a partir de 1919, con la vacuna humanizada. Domingo Orvañanos(1899)Ensayo de geografía médica, en Claudia Agostoni, "Estrategias, actores, promesas y temores en las campañas de vacunación antivariolosa en México: del porfiriato a la posrevolución (1880-1940)" en Ciencia \& Saude Colectiva, v.16, $\mathrm{n}^{\mathrm{o}} .2$, febrero 2011, p. 459-470. Asociacao Brasileira de Pos-Graduacao em Saude Colectiva. Río de Janeiro, Brasil.

${ }^{2}$ En algunas regiones la aplicación de la vacuna motivó una serie de enfrentamientos entre autoridades y habitantes de las comunidades rurales que no obstante no impidieron que hacia 1952 la viruela se declarara erradicada en México.
} 
profesores de diferentes especialidades, médicos, enfermeras, padres de familia y la comunidad rural en su conjunto ${ }^{3}$.

\section{LAS MISIONES CULTURALES: UN PROYECTO EDUCATIVO Y DE SALUD}

Durante el movimiento revolucionario en México (1910-1917), se puso en práctica una serie de proyectos educativos orientados a las necesidades de la población rural. La propuesta de la Escuela Rudimentaria (1913) no prosperó porque se reducía a la enseñanza de la lectura, la escritura y la aritmética ofreciendo a los niños alimentación y vestido para asegurar su asistencia. La Casa del Pueblo duro pocos meses a falta de presupuesto y de impacto en muchas de las regiones educativas del País. Al termino del movimiento revolucionario se inauguró la Secretaría de Educación Pública (1921) y con esto se diseñó un nuevo proyecto educativo, las Misiones Culturales, cuyo fin era resolver el aislamiento geográfico y la falta de presupuesto que habían mantenido, a la población indígena y campesina, al margen de las propuestas educativas del período anterior ${ }^{4}$.

Este aislamiento se mostraba en las estadísticas. Si bien los datos son ambiguos porque se mezclan diferentes criterios, sabemos que en el primer censo nacional general levantado en 1895, el $89 \%$ de la población vivía en el campo ${ }^{5}$. En el Censo de 1910 sólo el $11 \%$ de la población estaba clasificada como indígena y si atendemos al criterio de la lengua, cuatro quintas partes de los 15 millones de habitantes, hablaba español. Para 1921, el censo reportaba un 60\% de población mestiza y un 30\% de indígena, ${ }^{6}$ que para fines educativos representaba alrededor de 11 millones de indígenas y mestizos frente a un millón y medio de blancos. En este contexto había mucho trabajo por hacer, por esto, el nuevo proyecto educativo concibió al profesor como el líder de la escuela, encargado de promover los conocimientos y el desarrollo de habilidades en los niños y los adultos de la comunidad rural. Este cambio se observa claramente en la entrevista a Moisés Saenz, subsecretario de educación en 1924, en la que declaraba sus intensiones:

\footnotetext{
${ }^{3}$ Entendemos por modelo al conjunto de elementos y de relaciones entre estos diferentes elementos que "[...] presentan una coherencia interna mínima, una comprensión suficiente para reflejarse en el campo social y eventualmente un grado de legitimidad y formalidad para ser aceptado" (SAINT MARTIN, 2003, p. 10-11).

${ }^{4}$ Un proyecto educativo que ha sido caracterizado más como regenerador que como democratico (LISBONA GUILLÉN, 2006, p. 63).

${ }^{5}$ Fue sólo hasta la década de los sesenta del siglo XX que una parte de ésta fue considerada como urbana

${ }^{6}$ Para la discusión sobre estos datos véase, Castañeda, G., 2011, p. 142-145.
} 
[...] poco ganamos con enseñar a los niños solamente, 2 o 3 años de escuela son contrarrestados y anulados por el medio adulto, donde no se lee ni se escribe, ni se habla castellano, ni se tiene un ideal, ni se tiene una patria. Los niños y los adultos, toda la comunidad debe ser nuestra materia prima ... el maestro rural es el verdadero líder...

Moisés Sáenz había realizado estudios en la Sorbona de París y concluido un doctorado en Filosofía en la Universidad de Columbia donde preparó una tesis sobre la escuela secundaria. Como lo veremos en los apartados que siguen, influido por las ideas educativas del medio norteamericano, Sáenz integrará la propuesta de John Dewey en el proyecto de la escuela mexicana y se valdrá de la estructura del Young Men's Christian Association (YMCA) de la que será representante en México en estos años ${ }^{7}$.

Este proyecto llamado Misiones Culturales reunió a 4817 maestros rurales, 117 inspectores y un Director Federal por cada entidad (SECRETARÍA DE EDUCACIÓN PÚBLICA, 1927, p. 9). Cada misión formada por un equipo de profesores-misioneros se encargaron de viajar a diferentes lugares del país para realizar tres tareas primordiales: detectar los problemas sociales de la comunidad, actualizar la formación de los maestros rurales que se ocupaban de la escuela primaria y dar un conocimiento básico a los habitantes de los pueblos con saberes agrícolas para los hombres y de economía doméstica e higiene, para las mujeres ${ }^{8}$.

En 1926 se institucionalizó el programa y se creó la Dirección de Misiones Culturales que en unos años se transformó en Departamento de Enseñanza Agrícola y Normal Rural cuando se orientó hacia el mejoramiento de métodos y técnicas de la producción agrícola y se vincularon a las escuelas regionales campesinas y a las escuelas normales rurales. En 1938 el programa se canceló y cinco años después se restableció con nuevos objetivos y otra organización que lo modificaron sustancialmente (MENDOZA RAMIREZ, 2014, p. 11-15, 21).

\footnotetext{
${ }^{7}$ Moisés Saenz (1888 Mezquital, Monterrey-1941, Perú) estudió en la Escuela Preparatoria Presbiteriana de Coyoacán y en la escuela protestante. En la Universidad de Jefferson y Washington se especializa en Ciencias Químicas y Naturales y en la primera década de los años 20 se encarga de la Dirección General de Educación en el Distrito Federal, es Jefe del Departamento de Intercambio y de la dirección de la Escuela de Verano para Extranjeros. Hacia 1924 es Oficial Mayor de la SEP y un año después, Subsecretario de la SEP. Durante el proyecto de las Misiones Culturales apoyará las escuelas de pintura al aire libre, las escuelas especiales y los museos regionales. En su última etapa se integrará al Servicio Exterior Mexicano en Dinamarca, Ecuador y Perú. Interesado en el medio rural organiza el Congreso Indigenista en Pátzcuaro, Michoacán en abril de 1940 en el que se discute por primera vez la posibilidad de una enseñanza bilingüe (SÁENZ, 1992 y GONZÁLEZ GAMIO, 2003, p. 113-116).

${ }^{8}$ Cada Misión Cultural estaba formada por especialistas en higiene y educación física, agricultura, artesanías rurales, ciencias domésticas, carpintería y música; el jefe, a cargo de la Misión, era responsable de dar clases sobre métodos de enseñanza y organización, basados en las teorías pedagógicas modernas y en las necesidades locales. En la práctica la función de las misiones fue actualizar a los maestros y mejorar las condiciones higiénicas, económicas y culturales de los campesinos (RABY, 1974, p. 22-23).
} 


\section{LAS MISIONES CULTURALES Y LA INFLUENCIA NORTEAMERICANA}

El modelo de desarrollo físico norteamericano adquirió nuevos matices al adaptarse al medio rural mexicano. Se practicaron diferentes deportes y se intensificó el rescate del folklore, entendido como un bien cultural que preservaba la identidad. Hubo poblaciones que nunca habían visto las pelotas y tampoco conocían los llamados zapatos de hule que servían en la práctica de estos deportes ${ }^{9}$.

El modelo norteamericano se había inspirado del que se puso en práctica durante la Primera Guerra Mundial que a grandes rasgos podría explicarse de la siguiente manera. Francia e Inglaterra necesitaban mano de obra en razón de la movilización y las víctimas de guerra, para esto, se contrataron a cerca de 140 mil voluntarios chinos para suplir las vacantes. Los primeros barcos llegaron a Marsella y a Lyon, con trabajadores que fueron empleados en fábricas y puertos pero también para encargarse de las labores de limpieza en los frentes. En la integración de estos grupos asiáticos al medio europeo jugó un papel importante la Young Men's Christian Association (YMCA), una asociación cristiana, creada en el Reino Unido en 1844 que procuraba favorecer el desarrollo espiritual, intelectual, físico y social de los habitantes. Fueron instalados campamentos de trabajadores chinos en los que el YMCA organizó una serie de actividades deportivas y de entretenimiento. Por primera vez los trabajadores chinos asistieron a proyecciones de cine, conciertos, funciones de teatro, fiestas y actividades educativas porque el $80 \%$ de los trabajadores no sabía leer ni escribir y mucho menos el idioma. Se estimuló la asistencia a la iglesia los días domingo y la lectura de la biblia en reuniones en las que se ponía en práctica una educación impregnada de moral cristiana. Los manuales escolares y las revistas fueron un importante medio de difusión en estos centros porque condenaron los malos hábitos como el consumo de tabaco, los juegos de azar y la prostitución y se estimularon un sinfín de actividades deportivas como el basquetbol y las competencias deportivas. Los objetivos del YMCA estuvieron orientados principalmente a la difusión religiosa y civilizatoria pues "[...] una parte importante de su motivación era espiritual y desinteresada [...] en ayuda a los trabajadores $[\ldots] . " 10$.

\footnotetext{
${ }^{9}$ En esa época sólo existían en la Ciudad de México tres casas que se dedicaban a la venta de aparatos y ropa para usos deportivos: la Casa Pinedo, Artículos Deportivos Spaulding y la Casa Martí.

${ }^{10}$ Desde 1854 se fundó el International Committee of YMCA in USA and Canada que tuvo presencia en diferentes países. En China, en 1885 sus actividades se orientaron a la apertura de establecimientos escolares -liceos y 
Se trataba de un esquema que privilegiaba la práctica del deporte como antídoto para contrarrestar los efectos de la vida urbana pero además que servía como vehículo para transmitir una serie de valores asociados a la identidad estadounidense en un país que había recibido a cerca de 25 millones de inmigrantes entre 1865 y 1915. El proyecto se difundió para crear circuitos de competencia y de difusión. También se incorporó a los programas escolares de educación primaria y secundaria con lo que se permitió que los alumnos fueran instruidos en deportes como el beisbol, el basquetbol y el futbol americano, un espacio donde se ejercitaban valores de la vida democrática y se transmitían valores como la obediencia a las reglas, la cooperación, el reconocimiento a la excelencia (TORRES, 2011, p. 266).

Si bien el esquema del YMCA inicialmente tuvo un carácter de orden religioso fue también civilizatorio, y en países como México, Brasil y Uruguay, encontró amplia respuesta gracias a la variedad de actividades sociales y deportivas de su propuesta, porque se veían como el medio ideal para preparar al hombre para una nueva época en la que se promovía por igual el descanso y el trabajo (MARTINEZ MOCTEZUMA, 2016). A semejanza del caso asiático, el programa del YMCA que se implementa en las escuelas rurales en México puede resumirse en los siguientes aspectos: la formación de líderes sociales, la práctica del juego de conjunto y la habilidad para trabajar en cooperación con la comunidad. En este contexto, el ejercicio se convertía en una práctica social, donde la atención estaba puesta especialmente en el grupo y la dirección inteligente y preparada del maestro, así como en la acción coordinada de las actividades comunitarias.

\section{LA PRÁCTICA DEL EJERCICIO FÍSICO EN EL MEDIO RURAL}

Entre 1924 y 1926 se llevaron a cabo dos Misiones Culturales en el estado de Morelos, en las que se puso atención a la formación del maestro, de los alumnos y de la comunidad en general. Como era costumbre, al llegar a Cuautla y Puente de Ixtla, los integrantes de la misión eran recibidos por las autoridades municipales, estatales y federales. Después de la fiesta de

universidades- que estuvieron bajo la dirección de profesorados norteamericanos o de chinos que habían realizado estudios en Estados Unidos. En 25 años, entre 1895 y 1920, el YMCA aumentó el número de sus establecimientos escolares: 2 en 1901 y 30 en 1920, con 32 mil adherentes. El YMCA y sus misioneros en China tenían redes bien establecidas que fueron usadas para recrutar a los trabajadores chinos porque el War Office británico los reconoció como los propagadores perfectos de su filosofía: la propia población china les tenía respeto, afecto y confianza porque no eran vistos como corruptos (MA, 2009, p. 103-107). 
bienvenida, se inscribía a los asistentes y se establecía una cooperativa de alimentación cuyos fondos servían para el mantenimiento y la compra de material deportivo ${ }^{11}$.

Mapa 1. Estado de Morelos. México.

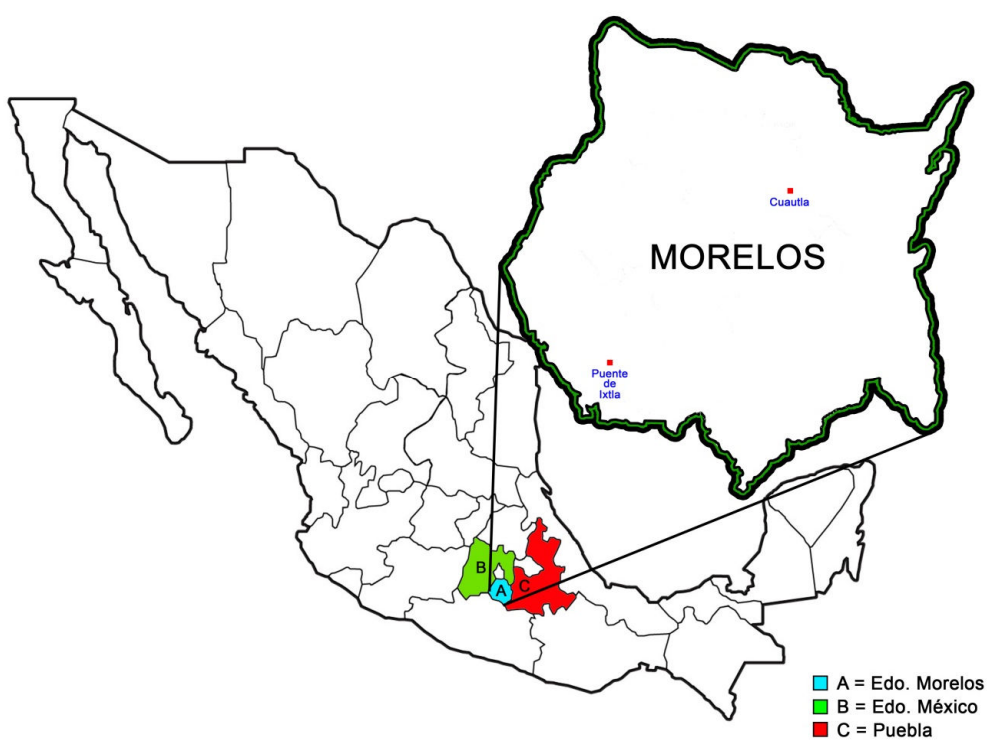

Fuente: En este mapa se encuentran señalados los municipios de Cuautla y Puente de Ixtla donde se realizaron las primeras Misiones Culturales en el estado de Morelos. Elaboración de Karina Patricia Ocampo.

En Cuautla, las clases para las maestras fueron programadas por la tarde, a partir de las 16:00 horas, mientras que a la misma hora, los hombres barbechaban y aplanaban el terreno en el que se realizarían las actividades físicas con la práctica del juego de beisbol, y colocaron canastas rústicas para la práctica del basquetbol ${ }^{12}$.

En este ambiente fue importante la participación de las mujeres y las niñas porque como lo ha señalado Mary Kay Vaughan (2000) eran ellas quienes colgaban las bandas de seda a los ganadores de los torneos y confeccionaban los uniformes deportivos pero también estuvieron presentes por primera vez en las actividades comunitarias. Las fotografías que acompañan los informes de maestros e inspectores localizados en el Archivo Histórico de la Secretaría de

\footnotetext{
${ }^{11}$ Martínez Moctezuma Lucía y Adan Guadarrama Adriana, "Los cursos de mejoramiento para profesores morelenses: un proyecto educativo del México posrevolucionario"

${ }^{12}$ Unos años después, en Puente de Ixtla, se anexó al mismo programa, una clase sobre estos deportes antes de la cena, con lo que se muestra que estas actividades empezaban a formar parte del currículo. Archivo Histórico de la Secretaría de Educación Pública (en adelante AHSEP) Caja 12, Exp.28, fj.11.
} 
Educación Pública, las muestra por primera vez entusiastas, sonrientes, participando tanto de las justas deportivas como de las reuniones dominicales acompañando a la familia ${ }^{13}$.

Fotografía 1. Mujeres participantes en las actividades de la Misión Cultural en Cuautla, Morelos.

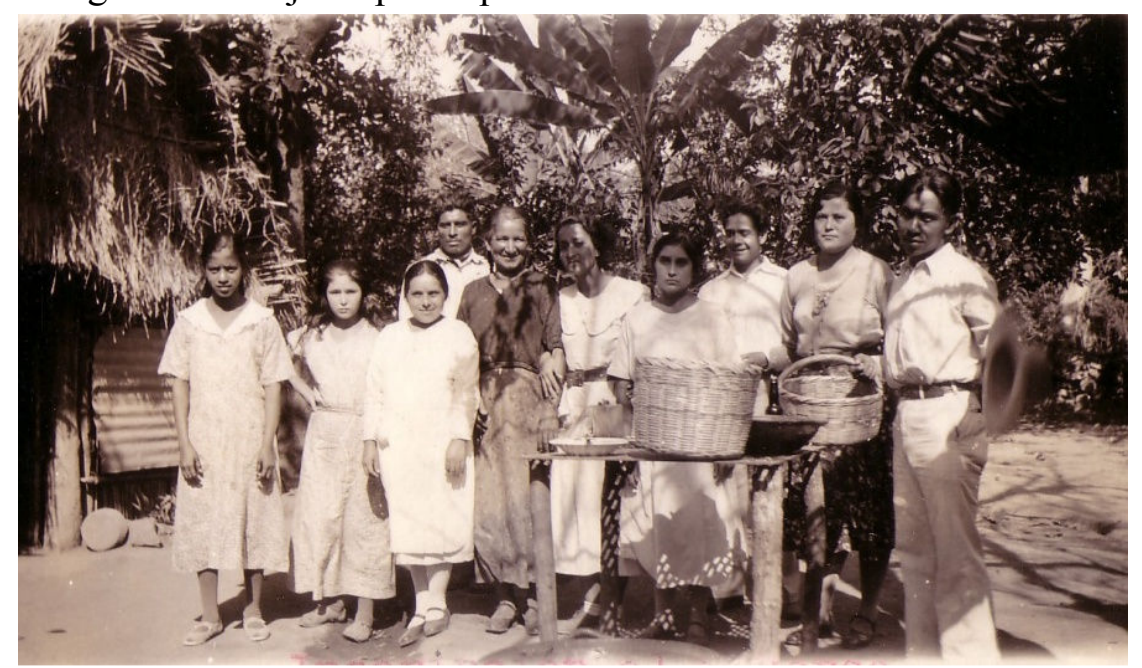

Fuente: AHSEP. Fondo Departamento de Enseñanza Agrícola y Normal Rural. Puente de Ixtla. Caja 4. Exp.14.

Como en otras entidades del país, los niños practicaban diariamente ejercicios de orden, tablas gimnasticas y juegos en grupo. Las actividades de todo el mes se clausuraban siempre con una fiesta en la que se programaba un encuentro atlético de ambos sexos con carreras de relevos, saltos de altura, y de longitud.

\footnotetext{
${ }^{13} \mathrm{Si}$ bien la práctica del deporte incorporó paulatinamente a la mujer no siempre fue vista como un paso a la igualdad de género. Un periódico en Chiapas reportaba que gracias a la práctica del basquetbol, “[...] deporte más gustado por las damitas [...] se nos mostraran el dia de mañana [...] con sus simpaticos pantalocitos y dispuestas a hacernos pasar un buen rato" en la Sección de Deportes del periódico El Sur de México, Tapachula, 4 de enero 1940 (citado en LISBONA, 2006, p. 81).
} 
Fotografía 2. Salto de altura en Cuautla, Morelos.

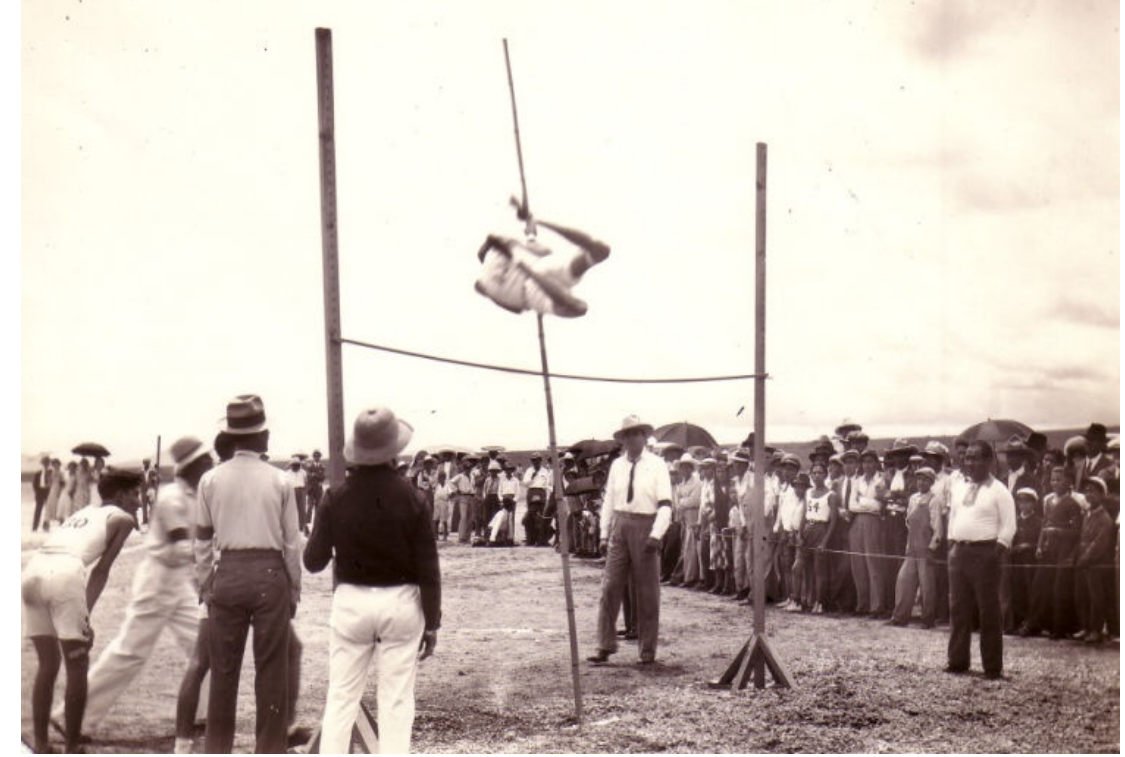

Fuente: AHSEP. Fondo Departamento de Enseñanza Agrícola y Normal Rural. Cuautla. Caja 4. Exp.2.

Algunos documentos de archivo señalan que la Misión Cultural se había instalado con el argumento de que se trataba de una región con poca oferta de trabajo y necesidades imperiosas de rehabilitación, por esto el objetivo principal de la misión cultural, apuntaba a la transformación social con la incorporación de la comunidad rural, particularmente de la indígena, a la vida moderna. Por esta razón se diseñaron una serie de estrategias para atender al mejoramiento de la vida doméstica, enfocándose hacia los aspectos material, económico, social y moral. Primero se hizo un estudio sobre la región, levantando un censo sobre la mortalidad infantil, después se analizaron las condiciones económicas e higiénicas de la familia, se contabilizó el mobiliario de cada hogar y se describieron minuciosamente los hábitos de la vida doméstica, alimentación, vestido, condiciones de trabajo de la familia, vida social y situación educativa. En los reportes de las autoridades se subraya la importancia del papel de la mujer en la práctica del deporte así como en el cuidado de la alimentación familiar.

Una vez que el equipo de trabajo de la misión cultural realizaba actividades dentro de la comunidad, el médico y la enfermera buscaban ganar la confianza de los padres de familia. El plan de trabajo de la enfermera estaba orientado hacia diferentes aspectos como la higiene general, el vestido, la alimentación, la práctica del ejercicio y el baño. También orientaban a las madres embarazadas y al recién nacido poniendo énfasis en el papel de la partera y la 
colaboración de la familia en el nacimiento. Cuando las enfermeras lograban ingresar a los domicilios, insistían en dar orientación sobre la enfermedad de los niños, sus síntomas y medidas preventivas. Otros temas fueron los del alcoholismo y las enfermedades de la región como el paludismo y otras que eran comunes a todo el país ${ }^{14}$. En cuanto al alcohol, se consideraba un vicio y se recomendaba el ejercicio físico con la práctica de deportes como el beisbol, el basquetbol y el futbol, sobretodo porque la comunidad gozaba de algunas horas libres los días domingo en los que podía fortalecerse la disciplina y la sociabilidad: formando diferentes grupos para despejar los terrenos que servían para las competencias, construían teatros al aire libre en los que se presentaban obras que informaban sobre la salud, la enfermedad y sobre todo el rechazo a las bebidas alcohólicas: "[...] participar de los eventos culturales y deportivos, era la mejor manera de [....] [alejarse)] de la cantina, de los gallos, y de los juegos prohibidos [...] [con lo cual] la moralidad va creciendo cada día" (MONROY, 1935, p. 126-128).

Estas pláticas no estuvieron exentas de conflictos y en muchas ocasiones los inspectores tuvieron que intervenir para señalar la colaboración que había entre el maestro y el médico pues el único fin era hacerles comprender a los padres de familia que la salud de los alumnos estaba entre sus deberes. Para la escuela, los padres de familia debían atender a las indicaciones del médico para prevenir o curar las enfermedades que se presentaran en sus hijos porque un niño aseado y sano representaba un orgullo para la familia pero también "una promesa para la patria" (AHSEP, Fondo Psicopedagogía e Higiene, 1930, p. 1-5).

Finalmente los encuentros deportivos siguieron siendo el medio ideal para rescatar las tradiciones, los bailes y los cantos locales, pero también, como lo señala Norbert Elías, para administrar el ocio porque se fomentaron hábitos para el cuidado del cuerpo y la práctica de buenas medidas higiénicas que alejaban al ciudadano de los malos vicios y el alcohol ${ }^{15}$.

\footnotetext{
${ }^{14}$ Además del paludismo se propagaban enfermedades como la viruela, la tiña, la sarna, los piojos, por esto se reivindicaba en la "Semana de la Salubridad" el empleo de la vacuna, jabón, baño y brigadas escolares" (P. NAÑEZ, 1928, p. 36).

${ }^{15}$ Quizá la falta de presupuesto, el cambio frecuente de personal y sobre todo, la puesta en práctica de un modelo de desarrollo físico, hizo que el profesor de educación física y Deportes declarara que en 1935, a su llegada al plantel, "[...] encontré un alumnado en un estado verdaderamente desastroso [...] por lo que hubo que esforzarse para trabajar aun con pequeñas deficiencias".
} 
Fotografía 3. Actividades deportivas y sociales presenciadas por la comunidad rural el día domingo.

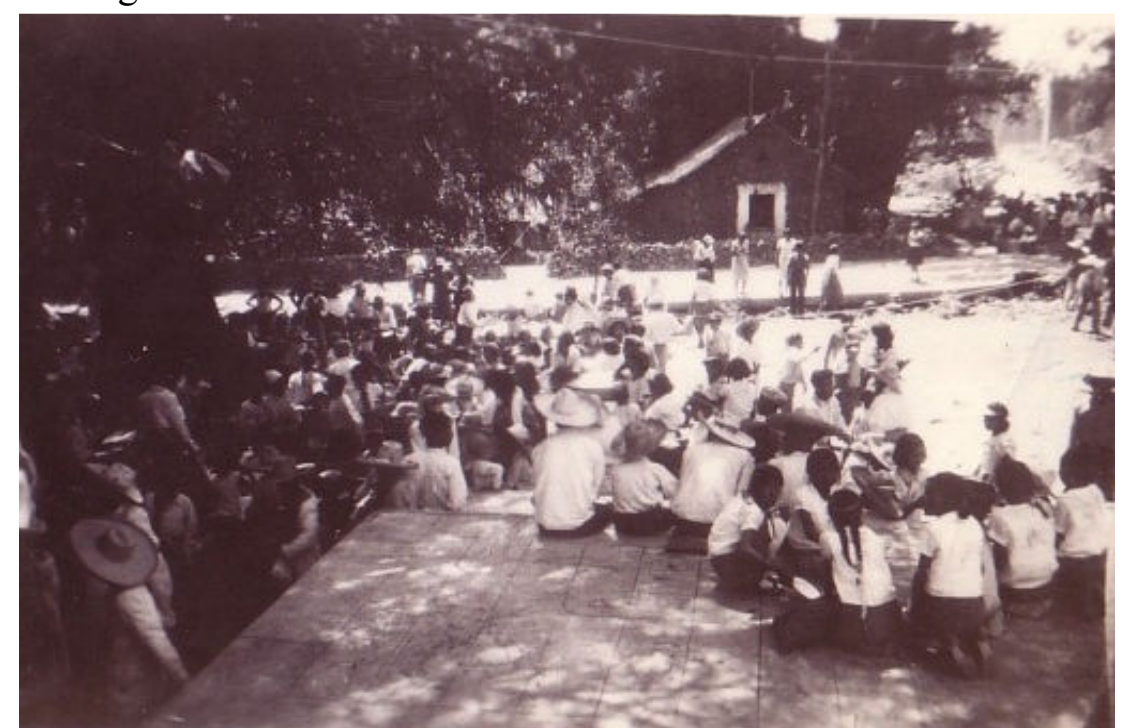

Fuente: AHSEP. Fondo Departamento de Enseñanza Agrícola y Normal Rural. Puente de Ixtla, Morelos. Caja 4. Exp.14.

Parecía una práctica difícil de erradicar porque como lo advertía en su informe el director, José Ramírez y el inspector, Arturo Arruti, era una tarea conjunta de maestros, alumnos, medicos $\mathrm{y}$ padres de familia, quienes procuraban planear y desarrollar programas adecuados que atendieran "la falta de comprensión de la población ante la tarea de la escuela, el fanatismo y la embriaguez...muy arraigados a consecuencia de la pobreza y la escasez de recursos ...". Por esto era importante que el médico y la enfermera obtuvieran la confianza de la comunidad, para lograr visitar e ingresar a los hogares, organizar pequeños festivales y funciones de cine donde pudieran socializar su proyecto. Los informes de los directores revelan también que su tarea había impactado por lo menos en el hecho de que las familias ya no dormían con los animales y se habían esmerado en el barrido y limpieza de las habitaciones, se había construido un cobertizo y un porqueriza, se armó un botiquín comunal y otro para la escuela, se estableció un gabinete de aseo y se encementó el piso del baño, varios adultos y niños habían acudido a cortarse el pelo y se logró que hicieran buen uso del agua potable y del baño por lo menos dos veces por semana. $\mathrm{Si}$ bien eran importantes estos cambios no podía evitarse que se presentara una epidemia de sarampion y se combatiera, "[...] la costumbre perniciosa de curarse con medicamentos inadecuados o creer en hechicerías o supersticiones malsanas [...]". 
Este modelo de actividades físicas impuesto en el siglo XX, fue consolidándose en diferentes entidades como el Estado de México, Quinta Roo, Tamaulipas, Puebla y Morelos que hicieron suya la propuesta norteamericana del desarrollo de actividades físicas, en las cuales fue primordial la participación de la comunidad rural. En Tampico, Tamaulipas se construyó especialmente el Estadio Victorense para reunir a los miembros de clubes deportivos, vecinos de la entidad y en cada manifestación deportiva se mostraba que: “[...] el pueblo entero se congrega, por hábito, domingo a domingo y en los días de grandes fiestas cívicas o escolares, a presenciar los diversos encuentros de base-ball, basket-ball, volley-ball, carreras, juegos libres $\mathrm{u}$ organizados, etc .” (P. NAÑEZ, 1928, p.42-34).

A semejanza de lo que sucedía en el caso de Puebla, las escuelas morelenses enseñaron menos en las escuelas y más en las fiestas y en los encuentros deportivos, porque como lo señala Vaughan (2000), se trató de una forma didáctica, social, política y, sobre todo, divertida, en la que los pueblos utilizaron el deporte y se apropiaron de las escuelas de acuerdo con sus necesidades, valores e intereses, un espacio en el que el maestro adoptó el papel de líder.

Hacia los años 40, con un nuevo proyecto educativo caracterizado como socialista, maestros e inspectores continuaron organizando eventos, "sencillos [...] a manera de pequeñas fiestas del pueblo", en los que se mostraba la destreza física de los niños y adultos con marchas, tablas gimnasticas, carreras, saltos, y si era posible, con el desarrollo de encuentros deportivos que fueron acompañados de música. Los informes de las profesores señalan también la existencia de ciertos grupos entre la población que minaban la labor de los equipos de trabajo y el descuido de parte de las autoridades que negaban todo tipo de apoyo.

\section{NOTAS FINALES}

La Revolución Mexicana de 1910 destruyó al Estado y se necesitaron tres décadas para edificar uno nuevo que fue construido por medio del dialogo con diversos movimientos sociopolíticos donde surgió una difícil negociación entre los actores en sitios regionales y locales en los que el poder se disputó y se desarrolló.

Durante los años 20, los jefes de la política regional se valieron de los profesores de la SEP y de la política cultural -la creación de símbolos revolucionarios, arte didáctico y teatro colectivo- para formar una base popular entre obreros y campesinos. En este contexto se explica 
como a partir de 1929 con la formación del Partido Nacional Revolucionario, la política cultural pasó a ser parte de la construcción de un partido nacional y la unificación del Estado (VAUGHAN, 2000 , p. 15).

La realización del Primer Congreso de Higiene Rural en 1935 muestra que hay un rezago en el número de médicos: 4250 médicos (1 500 para 70 ciudades, donde 600 servían a 13 millones de campesinos, a raíz de uno por 21 000habitantes) y que este vacío es ocupado por el maestro quien seguía siendo el mejor elemento para impartir educación higiénica en los medios rurales y como colaborador del médico a quien se les pedía que tomara cursos rápidos de higiene escolar. Finalmente se confirmaba que el equipo de trabajo formado por médicos, maestros rurales y autoridades era el indicado para mejorar la salud de ciudadano mexicano ${ }^{16}$.

\section{BIBLIOGRAFÍA}

AHSEP. Archivo Histórico de la Secretaría de Educación Pública. Fondo Psicopedagogía e Higiene. 1930.

AGOSTONI, Claudia. Estrategias, actores, promesas y temores en las campañas de vacunación antivariolosa en México: del porfiriato a la posrevolución (1880-1940). Ciencia \& Saude Colectiva, Asociacao Brasileira de Pos-Graduacao em Saude Colectiva. Río de Janeiro, Brasil, v.16, $\mathrm{n}^{\mathrm{o}} .2, \mathrm{p} .459-470$, febrero 2011.

CASTAÑEDA G., Jorge. Mañana o pasado. El misterio de los mexicanos. México: Aguilar, 2011.

CHÁVEZ GONZÁLEZ, Mónica Lizbeth. Los orígenes de la educación física en México. Reflexiones sobre el género, el cuerpo y la nación. México: BECENE, RIESLP, UNAM. Colección Investigadores y Maestros, 2015.

GALVÁN, Luz Elena. Maestros y escuelas rurales en la política educativa (1920-1940). En: OLIVA, Lesvia y CARRASCO, Rosas (coord.). La educación rural en México en el siglo XXI. México: CEE, CREFAL, 2007.

(coord). Miradas en torno a la educación de ayer. Investigación educativa 1993-1995. México: COMIE-UdeG, 1997.

GONZÁLEZ GAMIO, Ángeles. Manuel Gamio una lucha sin final. México: UNAM, 2003.

${ }^{16}$ Primer Congreso Nacional de de Higiene Rural celebrado en Morelia, 3 al 12 de noviembre de 1935, p. 303. Rev. Iberoam. Patrim. Histórico-Educativo, Campinas (SP), v. 2, n. 3, p. 101-116, jul./dez. 2016 
LISBONA GUILLÉN, Miguel. Apuntes sobre la práctica deportiva en Chiapas (1910-1940). Pueblos y Fronteras Digital, Universidad Nacional Autónoma de México, n. 2, 2006.

LOYO, Engracia. En el aula y la parcela: vida escolar en el medio rural (1921-1940). En: DE LOS REYES, Aurelio (coord.). Historia de la vida cotidiana en México, v. 5. México: Siglo XX. Campo y ciudad, FCE, COLMEX, 2006.

MA, Li. La place de la religion dans l'éducation par le YMCA des travailleurs chinois de la Grand Guerre. Guerres Mondiales et conflits contemporains, v. 3, n. 235, 2009. Disponible en: $<$ https://www.cairn.info/revue-guerres-mondiales-et-conflits-contemporains-2009-3-page-101.htm>. Consultado en: septiembre 2015.

MARTÍNEZ MOCTEZUMA, Lucía. Escuelas normales de Cuernavaca y Oaxtepec, 1889-1933. Inventio. La génesis de la Cultura Universitaria en Morelos, año 1, n. 2, p. 13-22, septiembre 2005.

MENDOZA RAMÍREZ, Martha Patricia. Las Misiones Culturales y la escuela rural en Quintana Roo, 1927-1934. México: CIESAS. Publicaciones de la Casa Chata, 2014.

MONROY, H. El Agrarista. Libro $2^{\circ}$ de lectura para uso de las escuelas nocturnas rurales. México: Editorial Patria, 1935.

NORBERT, Elías y DUNNING, Eric. Deporte y ocio en el proceso de la civilización. México: Fondo de Cultura Económica, 1992.

P. NAÑEZ, Maurilio. Renovación. Tópicos del Estado de Tamaulipas desarrollados durante el gobierno del Sr. Emilio Portes Gil. Tamaulipas: Editora Coahuilense S.A., 1928.

RABY, David. Educación y revolución social en México. 1921-1940. México: Secretaría de Educación Pública, 1974.

SANTIAGO, Sierra. Las Misiones Culturales (1923-1973). México: SepSetentas, 1993.

SÁENZ, Moisés. Carapan. México: Centro de cooperación regional para la educación de Adultos en América Latina y el Caribe. CREFAL, 1992.

SAINT-MARTIN, Jean. Education Physique françaises et exemplarités étrangères entre 1815 et 1914. Francia: L'harmattan, 2003.

SECRETARIA DE EDUCACIÓN PÚBLICA. Las misiones culturales en 1927. Las escuelas normales rurales. México: SEP, 1928.

TORRES, César. La educacion física en Estados Unidos (1865-1945). En: SCHARADRODSKY, Pablo. La invención del "homo gymnasticus". Fragmentos históricos sobre educación de los cuerpos en movimiento en Occidente. Argentina: Prometeo Libros, 2011. 
VAUGHAN, Mary Kay. La política cultural en la revolución. Maestros, campesinos y escuelas en México. México: Secretaría de Educación Pública, Fondo de Cultura Económica, 2000.

VÉLAZQUEZ, Andrade Manuel. Conferencia téorico práctica sobre educación física dada a los delegados del $2^{\circ}$ Congreso Nacional de Educación Primaria el sábado 30 de septiembre de 1911 en el gimnasio de la Esciuela Normal primaria para maestros, México Imprenta de A.Carranza e hijos.

V. V. AA. Los maestros y la cultura nacional, 1920-1952. México: SEP, Museo Nacional de Culturas populares, vol.3. Centro, 1987. 